\title{
Collimated synchrotron threads linking the radio lobes of ESO 137-006
}

\author{
M. Ramatsoku ${ }^{1,2}$, M. Murgia ${ }^{2}$, V. Vacca ${ }^{2}$, P. Serra ${ }^{2}$, S. Makhathini ${ }^{1}$, F. Govoni ${ }^{2}$, O. Smirnov ${ }^{1,3}$, L. A. L. Andati ${ }^{1}$, \\ E. de Blok ${ }^{7,5,6}$, G. I. G. Józsa ${ }^{3,1,4}$, P. Kamphuis ${ }^{9}$, D. Kleiner ${ }^{2}$, F. M. Maccagni ${ }^{2}$, D. Cs. Molnár ${ }^{2}$, A. J. T. Ramaila ${ }^{3}$, \\ K. Thorat ${ }^{8}$, and S. V. White ${ }^{1}$
}

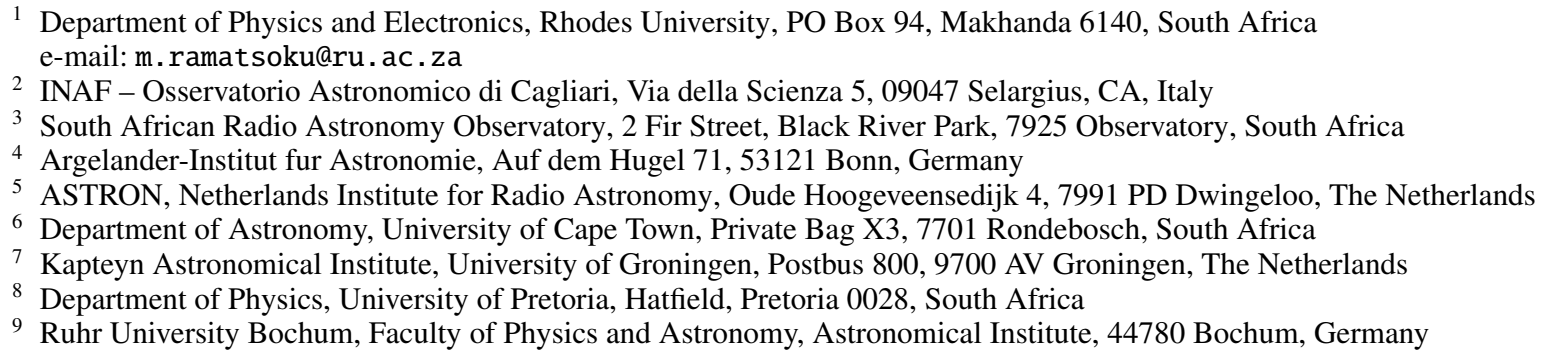

Received 23 February 2020 / Accepted 9 March 2020

\section{ABSTRACT}

\begin{abstract}
We present MeerKAT $1000 \mathrm{MHz}$ and $1400 \mathrm{MHz}$ observations of a bright radio galaxy in the southern hemisphere, ESO 137-006. The galaxy lies at the centre of the massive and merging Norma galaxy cluster. The MeerKAT continuum images $\left(\mathrm{rms} \sim 0.02 \mathrm{mJy} \mathrm{beam}^{-1}\right.$ at $\sim 10^{\prime \prime}$ resolution) reveal new features that have never been seen in a radio galaxy before: collimated synchrotron threads of yet unknown origin, which link the extended and bent radio lobes of ESO 137-006. The most prominent of these threads stretches in projection for about $80 \mathrm{kpc}$ and is about $1 \mathrm{kpc}$ in width. The radio spectrum of the threads is steep, with a spectral index of up to $\alpha \simeq 2$ between 1000 and $1400 \mathrm{MHz}$.
\end{abstract}

Key words. radio continuum: galaxies

\section{Introduction}

Radio galaxies residing in galaxy clusters exhibit a wide range of distorted morphologies (see Garon et al. 2019 for an overview). Some of these distortions are seen in the form of head tails, which are elongated radio sources with the galaxy at one end (Ryle \& Windram 1968; Sebastian et al. 2017), and wide-angle tails, where the jets form a "C" shape (Owen \& Rudnick 1976; Leahy 1993; Missaglia et al. 2019). Such radio morphologies are due to the interaction between the radio lobes and/or jets and the intra-cluster medium (ICM; Pinkney 1995; Sakelliou \& Merrifield 2000). This makes radio galaxies important probes of the distribution of pressure, turbulence, and shocks within the magneto-ionic ICM (Owen et al. 2014; Feretti et al. 2012), and also means that their detection is an efficient way to identify high- $z$ clusters independent of dust extinction.

In this Letter we discuss the case of ESO 137-006, a radio galaxy in the Norma galaxy cluster (Abell 3627; Abell et al. 1989). Norma is located at a distance of $\sim 70 \mathrm{Mpc}(z=0.0162$; Woudt et al. 2008) at the crossing between several filaments in the Great Attractor region (Dressler et al. 1987). With a dynamical mass of $\sim 10^{15} M_{\odot}$, Norma is characterised by numerous substructures and exhibits an elongated X-ray morphology, implying that it is not yet in dynamical equilibrium (Boehringer et al. 1996; Woudt et al. 2008).
ESO 137-006 $\left(\mathrm{RA}_{J 2000}=16: 15: 03.8, \operatorname{Dec}_{J 2000}=-60: 54: 26\right)$ is one of the most luminous galaxies in the Norma cluster and lies near the peak of the X-ray emission. It is one of the brightest radio galaxies in the southern sky $\left(L_{1.4 \mathrm{GHz}}=2.5 \times 10^{25} \mathrm{~W} \mathrm{~Hz}^{-1}\right.$; Sun 2009). Observations at $408 \mathrm{MHz}$ (Schilizzi \& McAdam 1975), $843 \mathrm{MHz}$ (Mauch et al. 2003), and $1400 \mathrm{MHz}$ (Christiansen et al. 1977; Jones \& McAdam 1996) show that it has a wide-angle tail morphology. The bending of its radio lobes is thought to be caused by the galaxy infall towards the main cluster (Jones \& McAdam 1996; Sakelliou \& Merrifield 2000). In this Letter we present new radio continuum images of ESO 137-006 at $1000 \mathrm{MHz}$ and $\sim 1400 \mathrm{MHz}$ based on MeerKAT observations, which reveal hitherto unseen collimated synchrotron threads (CSTs) between its radio lobes.

Throughout this paper we assume a $\Lambda$ cold dark matter cosmology with $\Omega_{\mathrm{M}}=0.3$ and $\Lambda_{\Omega}=0.7$, and a Hubble constant of $H_{0}=70 \mathrm{~km} \mathrm{~s}^{-1} \mathrm{Mpc}^{-1}$. At the distance of ESO 137-006, 1" corresponds to $0.33 \mathrm{kpc}$.

\section{MeerKAT observations and data reduction}

We observed the Norma cluster at radio frequencies with MeerKAT (Jonas \& MeerKAT Team 2016; Mauch et al. 2020) in May 2019 (project ID SCI-20190418-SM-01). The observations 
were conducted with all 64 MeerKAT antennas in $L$-band (856-1712 MHz) using the $4 \mathrm{k}$ mode of the SKARAB correlator, which samples the observed band with 4096 channels, each of $209 \mathrm{kHz}$ in width, in full polarisation. The total integration time is $14 \mathrm{~h}$ on target.

We reduced the data in two frequency intervals largely free of radio-frequency interference: $980-1080 \mathrm{MHz}$ and $1356-$ $1440 \mathrm{MHz}$ (hereafter referred to as $1030 \mathrm{MHz}$ and $1398 \mathrm{MHz}$, respectively). The data reduction was conducted independently in the two intervals using the CARACAL pipeline currently under development ${ }^{1}$. The pipeline is built using STIMELA ${ }^{2}$ (Makhathini 2018), a radio interferometry scripting framework based on Python and container technologies. STIMELA allows users to run several open-source radio interferometry software packages in the same script. Using this pipeline, we flagged the calibrator data based on the Stokes $Q$ visibilities with AOFLAGGER (Offringa et al. 2010). We determined the complex flux, bandpass, and gains using the CASA (McMullin et al. 2007) tasks bandpass and gaincal, and applied the calibration to the visibilities of the target with the CASA task mstransform. The calibrated target visibilities were flagged with AOFLAGGER, again based on Stokes $Q$. We then iteratively imaged the radio continuum emission with WSCLEAN (Offringa et al. 2014) in Stokes $I$ using multi-scale cleaning (Offringa \& Smirnov 2017), and selfcalibrated the gain phase with CUBICAL (Kenyon et al. 2018) with a solution interval of $128 \mathrm{~s}$. The imaging was done using Briggs robust value 0 and cleaning down to $0.5 \sigma$ within a clean mask made with SoFIA (Serra et al. 2015). Finally, we generated MeerKAT primary beam images at the mean frequency of the two processed bands using EIDOS (Asad et al. 2019), and created primary beam-corrected continuum images of the target.

The resulting $1030 \mathrm{MHz}$ image has a restoring beam of $10.0^{\prime \prime} \times 9.1^{\prime \prime}$ FWHM with PA $=169^{\circ}$, and $\mathrm{rms}$ noise level $30.8 \mu \mathrm{Jy}$ beam $^{-1}$. The $1398 \mathrm{MHz}$ image has a restoring beam of $7.5^{\prime \prime} \times 6.8^{\prime \prime}$ FWHM with PA $=167^{\circ}$, and rms noise level $20.8 \mu \mathrm{Jy}_{\text {beam }}{ }^{-1}$.

\section{ESO 137-006 as seen by MeerKAT}

Figure 1 shows the $1030 \mathrm{MHz}$ MeerKAT image of ESO 137-006. Emission shown in red to yellow is known from previous, shallower observations obtained with other telescopes (see Sect. 1). Emission shown in grey scale is revealed here for the first time due to the increased sensitivity and resolution of the MeerKAT data. A number of new features are now apparent.

Similar to earlier observations, we detect a point source corresponding to the galaxy ESO 137-006 (Jones \& McAdam 1996). At the resolution of our images this source has a flux density of $140 \mathrm{mJy}$ at $1030 \mathrm{MHz}$ and $167 \mathrm{mJy}$ at $1398 \mathrm{MHz}$. The source is also seen in X-rays (see Fig. 3) and is thought to be the core of ESO 137-006 (e.g., Jones \& McAdam 1996). Narrow jets start from the core along an east-west axis and form two broad, bright spots $\sim 1^{\prime}$ from it. Their peak flux densities are on average $\sim 8 \mathrm{Jy} \mathrm{beam}^{-1}$ at $1030 \mathrm{MHz}$ and $\sim 18 \mathrm{Jy} \mathrm{beam}^{-1}$ at $1398 \mathrm{MHz}$. Jones \& McAdam (1996) suggested that the brightening and expansion of the jets into these bright spots is due to the decreased ISM pressure. The general structure of these bright spots (see also Fig. 2) is similar to that seen in the hydrodynamical simulations of jets in the FR I radio galaxy 3C 31, where the jets recollimated at $\sim 1.5 \mathrm{kpc}$ from the galaxy core due to the

\footnotetext{
1 https://caracal . readthedocs.io

2 https://github.com/SpheMakh/Stimela
}

decreased pressure relative to the ISM after their initial expansion (Perucho \& Martí 2007).

Further out, the jets flare into the lobes and then fade into diffuse emission. On both sides, the radio emission bends southwards; in the western lobe the bending occurs right after the bright spot while in the eastern lobe it occurs further out.

Besides the previously known features described immediately above, Fig. 1 shows additional, low-surface-brightness radio emission at the edge of both lobes and, most strikingly, a number of CSTs in the region south of the core. These extend from the lobes southward of the core. Figure 2 shows the gradient image obtained with a Sobel convolution kernel that highlights the presence of three threads: CST1, CST2, and CST3. These threads are reminiscent of twin jets originating from two nuclei associated with radio source 3C 75 (Owen et al. 1985), except for the fact that in the case of ESO 137-006 only one nucleus is seen. Figure 1 also shows a diffuse loop of emission which starts at the base of CST3, extends around the eastern lobe on the south side, and connects back to the lobe on its easternmost side where it fades into diffuse emission. The origin of these features is not known. It is possible that these loops and CSTs are the result of some electromagnetic effect like parallel magnetic field lines within the ICM dragging particles between the lobes as the galaxy moves north, perhaps similar to the description by Heyvaerts \& Norman (1989).

The spectral index image $\left(S_{v} \propto v^{-\alpha}\right)$ of ESO 137-006 between 1030 and $1398 \mathrm{MHz}$ is presented in the top panel of Fig. 3. We find a spectral index distribution typical of an active wide-angle tail radio source. The radio core has a flat spectral index $\alpha \simeq 0$ while the inner and brighter parts of the jets have $\alpha \simeq 0.5-0.6$. There is a steepening in the radio spectrum going from the radio lobes down to the tails where we measure a spectral index as high as $\alpha \simeq 4$. The spatial variation of the spectral index in the eastern lobe is more gradual than in the western lobe, where a sharp transition, likely due to projection effects, is observed between the edge of the lobe and the underlying tail emission. The newly discovered CSTs are characterised by a steep radio spectrum with $\alpha \simeq 2$.

In the bottom panel of Fig. 3 we present the MeerKAT image at $1030 \mathrm{MHz}$ superimposed on the X-ray emission map from $X M M-N e w t o n$ at $0.5-2 \mathrm{keV}$. The most relevant feature seen in the $\mathrm{X}$-ray image is a point-like source associated with the radio core and a hint of cavities corresponding to the radio lobes, although no evidence for cavities associated with this source is present in the literature (Shin et al. 2016). At the sensitivity limit of the $\mathrm{XMM}$ image, there are no X-ray features that we can associate with these CSTs.

\section{Basic properties of CST1}

Figure 4 shows the deconvolved full width half maximum $\left(F W H M_{\text {dec }}\right)$ and the peak brightness $\left(I_{\text {peak }}\right)$ as a function of position along CST1 at 1030 and $1398 \mathrm{MHz}$, as well as the spectral index $\left(\alpha_{1030 \mathrm{MHz}}^{1398 \mathrm{MH}}\right)$. We measure these quantities at the positions shown by the red bars in the inset. The cross in the inset corresponds to the origin of the plot's horizontal axis. Errors were computed using a Monte Carlo simulation with input parameters sampled and noised in the same way as for the real data. The errors represent the scatter of the input versus output best-fit parameters. The mean values of the peak brightness are respectively $I_{\text {peak, } 1030 \mathrm{MHz}}=(8 \pm 1) \mathrm{mJy} \mathrm{beam}^{-1}$ and $I_{\text {peak, } 1398 \mathrm{MHz}}=(4.3 \pm 0.6) \mathrm{mJy}$ beam $^{-1}$, and the average spectral index $\alpha_{1030 \mathrm{MHz}}^{1398 \mathrm{MHz}}=1.8 \pm 0.3$, consistent with the values shown in Fig. 3. 


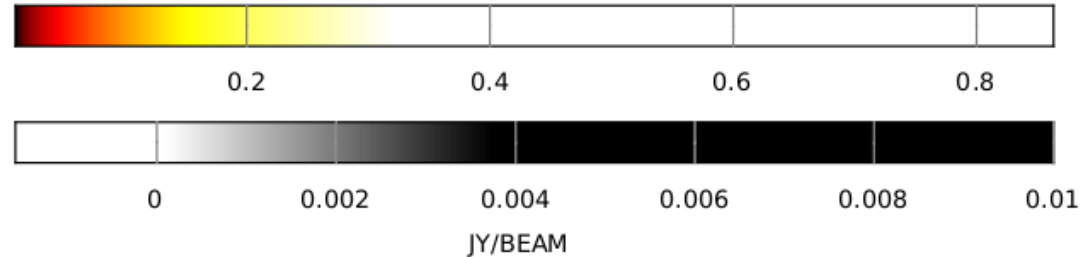

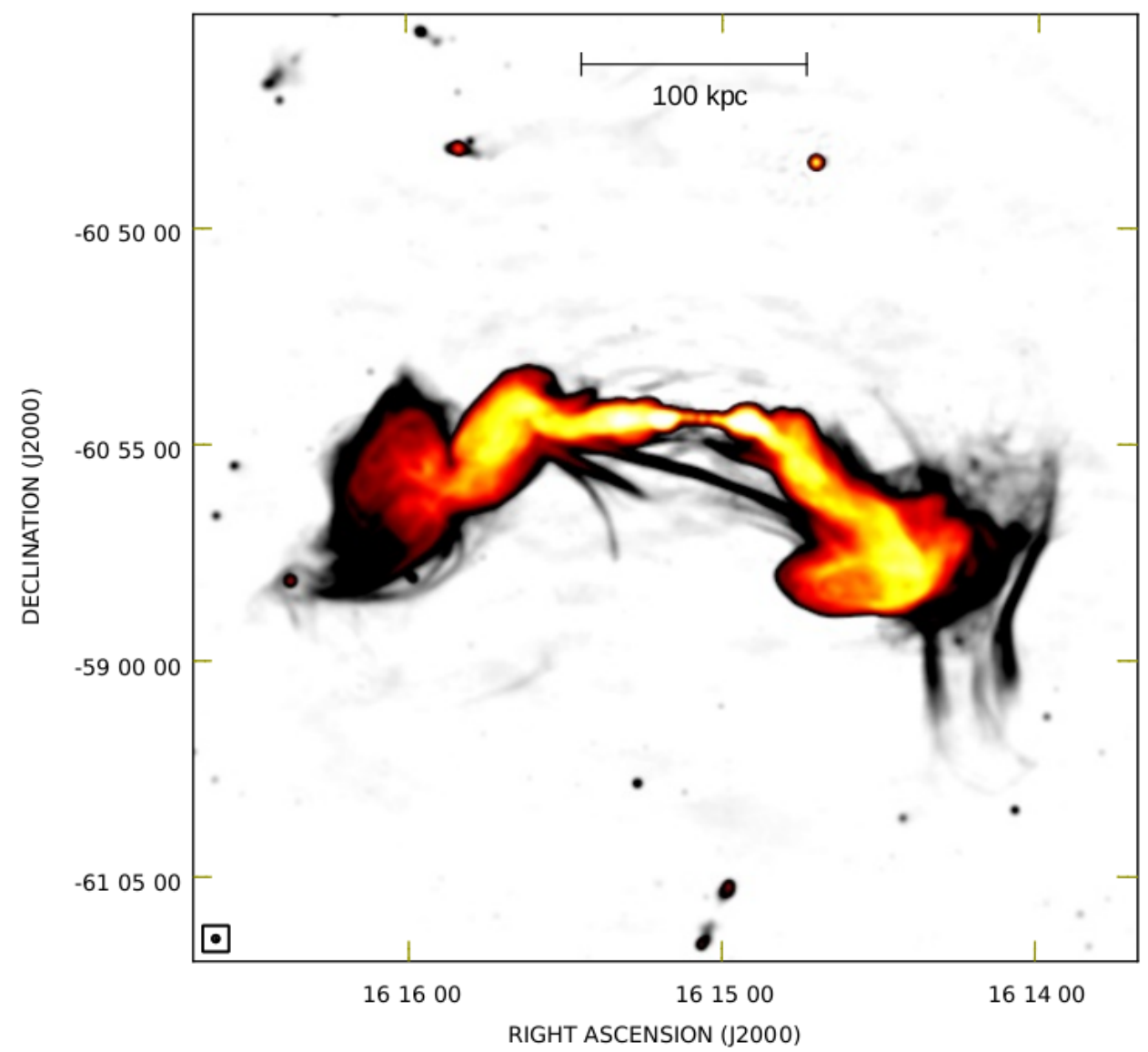

Fig. 1. Radio continuum emission from ESO 137-006 detected by MeerKAT at $1030 \mathrm{MHz}$. The top colour bar (yellow and red tones) represents the brightness of the brighter regions of the radio source in the range from 10 to $860 \mathrm{mJy}$ beam $^{-1}$. The bottom colour bar (grey tones) represents the brightness of the fainter plumes and filaments in the range from 10 down to $-1.6 \mathrm{mJy}_{\text {beam }}{ }^{-1}$. The circular $\sim 10^{\prime \prime}$ synthesised beam of the image is shown in the bottom left corner.

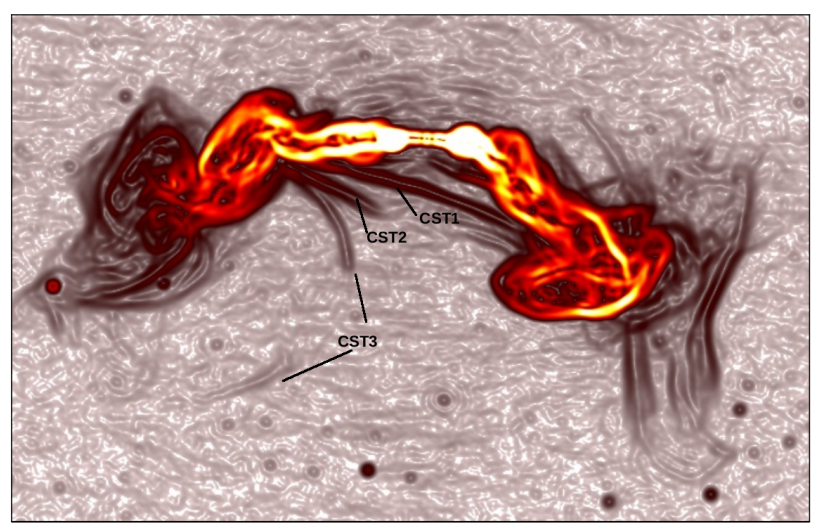

Fig. 2. Gradient image (Sobel convolution kernel) with the three most prominent CSTs labelled.

The peak brightness shows a similar profile at both frequencies, with a valley at $\sim 60^{\prime \prime}$ at $1030 \mathrm{MHz}$ only, which corresponds to a depression in the spectral index profile. At the same location, a rapid local increase of the deconvolved FWHM can be observed at both frequencies. The mean width of CST1 is $F W H M_{\mathrm{dec}, 1030 \mathrm{MHz}}=(3.5 \pm 1.6)^{\prime \prime}$ and $F W H M_{\mathrm{dec}, 1398 \mathrm{MHz}}=$ $(3.5 \pm 1.2)^{\prime \prime}$. The two profiles do not show any dependence on frequency but are rather characterised by a similar average trend along the full extension of the filament, consistent within the scatter. This projected width translates into $1.2 \pm 0.5 \mathrm{kpc}$, or $\sim 2 \%$ of its $80 \mathrm{kpc}$ length.

\section{Discussion and summary}

In this Letter we present new MeerKAT images of the radio source ESO 137-006 at 1000 and $1400 \mathrm{MHz}$. The galaxy lies at the centre of the merging Norma cluster near the Great Attractor. Here we summarise our main findings:

- With these sensitive MeerKAT observations, new features have been revealed in the form of multiple collimated synchrotron threads (CSTs) connecting the lobes of the radio galaxy. It is worth noting that examples of filamentary structures associated with radio galaxies are well known in the literature. However, these filaments are usually observed inside the radio lobes (see e.g. the notable cases of Fornax A and Cygnus A; Maccagni et al. 2020; Perley et al. 1984) and the tails of radio galaxies (e.g., NGC 1265, 3C 129, and NGC 326; Sijbring \& de Bruyn 1998; Lane et al. 2002; Hardcastle et al. 2019). The CSTs detected in ESO 137-006 are different in that they are observed outside the main body of the radio galaxy and connecting (at least in projection) the 

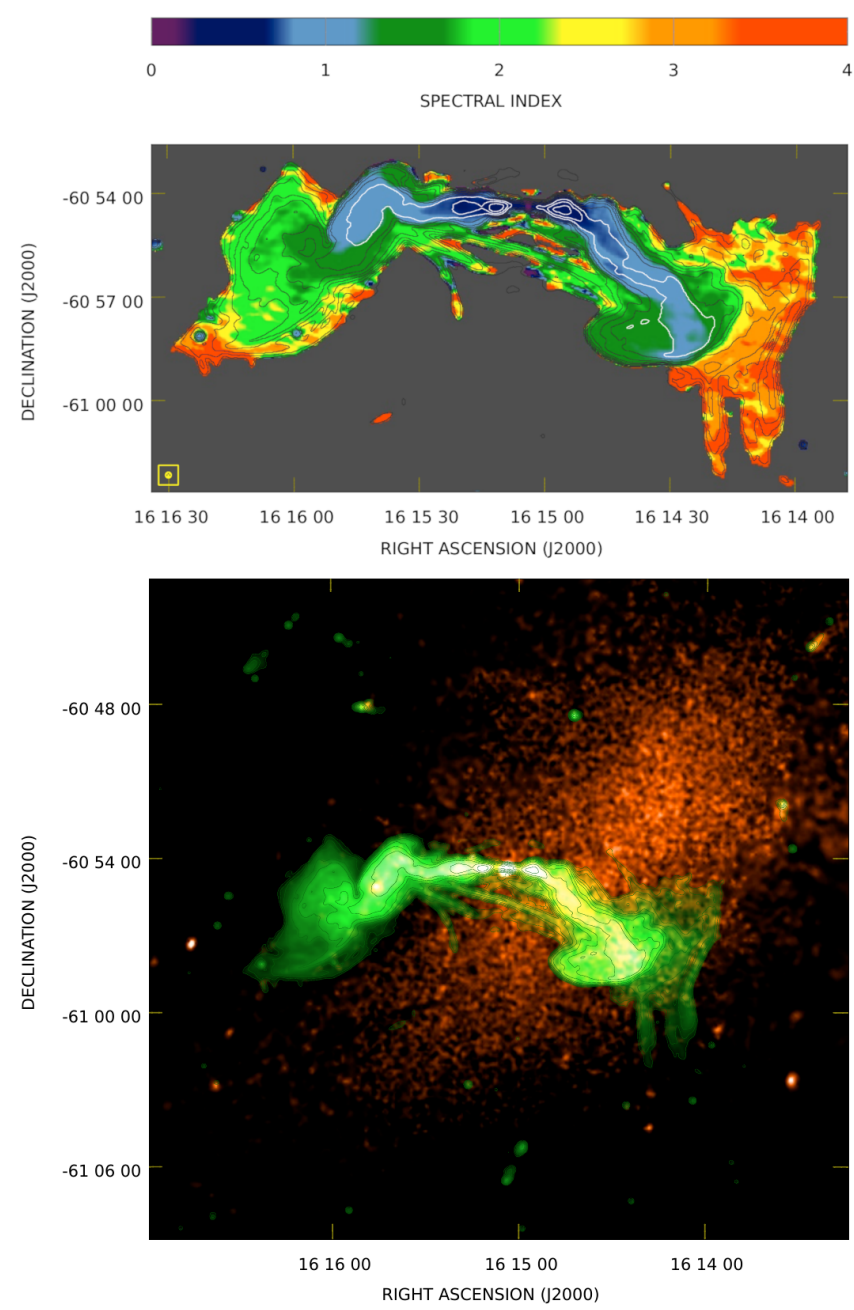

Fig. 3. Top panel: spectral index image between 1030 and $1398 \mathrm{MHz}$. Bottom panel: radio continuum emission from ESO 137-006 detected by MeerKAT at $1030 \mathrm{MHz}$ (green tones and contours) superimposed on the X-ray emission map from XMM-Newton at 0.5-2 keV. In both panels, radio contours refer to the $1030 \mathrm{MHz}$ image; they start at $0.5 \mathrm{mJy}^{\text {beam }}{ }^{-1}$ and scale by a factor of two.

two radio lobes. The radio galaxy 3C 338 (Burns et al. 1983) at the centre of Abell 2199 presents a single filament that is reminiscent of one of the CSTs observed in ESO 137-006. However, while the filament in 3C 338 could be a relic jet from a past epoch of activity, this same interpretation does not hold for ESO 137-006 where we observe multiple closeby threads formed at the same time (as suggested by their similar spectral-index distributions).

- The most prominent and straight of the CST in ESO 137-006 (CST 1) has a characteristic width of $\sim 1 \mathrm{kpc}$ (deconvolved FWHM), roughly $2 \%$ of its length, and has a relatively smooth brightness profile with a peak intensity of a few mJy beam ${ }^{-1}$ at the $10^{\prime \prime}$ resolution of our images. The other two CSTs originate from the same point in the eastern lobe. CST 2 starts straight and then fades rapidly after $\sim 25 \mathrm{kpc}$ from the lobe. CST 3 seems to follow a faint, closed loop with a radius of $\sim 64 \mathrm{kpc}$, which reconnects with the lobe at its far end. The nature of these unusual features is unclear. We speculate that they could be due to the interaction of the magnetic fields of the radio lobes with the magneto-ionic ICM, or caused by some sort of re-connection of filaments

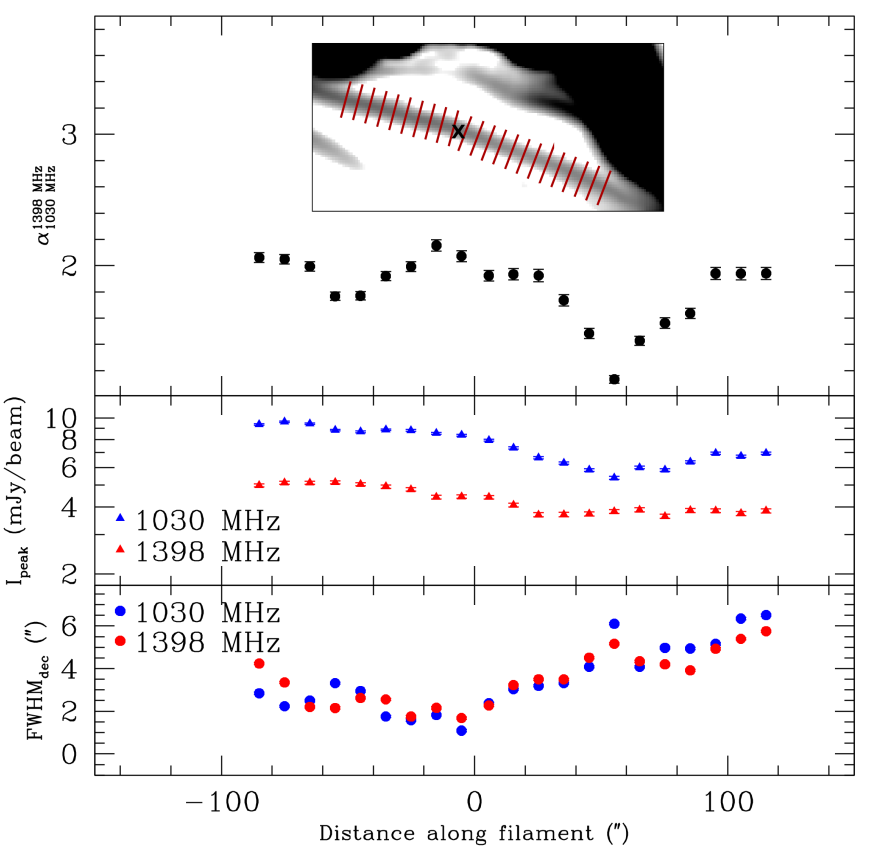

Fig. 4. Top panel: spectral index profile between 1030 and $1398 \mathrm{MHz}$ computed along the filament at the locations shown by the slices in the inset (see text for details). Middle panel: peak brightness at $1030 \mathrm{MHz}$ (blue triangles) and at $1398 \mathrm{MHz}$ (red triangles) along the filament at the location of the slices. Bottom panel: deconvolved FWHM at $1030 \mathrm{MHz}$ (blue dots) and at $1398 \mathrm{MHz}$ (red dots) along the filament at the location of the slices. The plotted error bars are comparable to the size of the dots.

associated with the tails back into the radio lobes. Further observations and theoretical efforts are required to clarify the nature of these newly discovered features.

- The spectral index distribution observed across the jets, lobes, and tails is typical of an active radio source. The radio spectrum of the CST is steep with $\alpha \simeq 2$. Due to this steep spectrum, deep low-frequency observations at high resolution with instruments such as the LOw Frequency ARray could play a role in the study of CSTs.

- Whatever their origin, our findings pose the following questions: How common are these features? Are CSTs specific to the case of ESO 137-006 and its environment, perhaps due to the kinematics and pressure gradient of this ICM and relative motion of the galaxy in the cluster? Or, on the contrary, are CSTs common in radio galaxies but have so far not been detected due to sensitivity and resolution limits? If future observations confirm the latter hypothesis, understanding the nature and the physics of these features could open a new science case for the next generation of sensitive radio interferometers like the Square Kilometre Array.

Acknowledgements. We thank the referee, Manel Perucho for the useful comments and suggestions. The authors also wish to thank Ming Sun for helping with the access to some of the X-ray data. This project has received funding from the European Research Council (ERC) under the European Union's Horizon 2020 research and innovation programme grant agreement no. 679627 project name FORNAX. MR acknowledges support from the Italian Ministry of Foreign Affairs and International Cooperation (MAECI Grant Number ZA18GR02) and the South African Department of Science and Technology's National Research Foundation (DST-NRF Grant Number 113121) as part of the ISARP RADIOSKY2020 Joint Research Scheme. This paper makes use of the MeerKAT data (Project ID: SCI-20190418-SM-01). The MeerKAT telescope is operated by the South African Radio Astronomy Observatory, which is a facility of the National Research Foundation, an agency of the Department of Science 
and Innovation. MR and SM's research is supported by the SARAO HCD programme via the "New Scientific Frontiers with Precision Radio Interferometry" research group grant. This work is based upon research supported by the South African Research Chairs Initiative of the Department of Science and Technology and National Research Foundation. (Part of) the data published here have been reduced using the CARAcal pipeline, partially supported by BMBF project 05A17PC2 for D-MeerKAT. Information about CARAcal can be obtained online under the https://caracal.readthedocs.io/en/latest/.

\section{References}

Abell, G. O., Corwin, H. G., Jr, \& Olowin, R. P. 1989, ApJS, 70, 1 Asad, K. M. B., Girard, J. N., de Villers, M., et al. 2019, MNRAS, submitted [arXiv:1904.07155]

Boehringer, H., Neumann, D. M., Schindler, S., \& Kraan-Korteweg, R. C. 1996 ApJ, 467, 168

Burns, J. O., Schwendeman, E., \& White, R. A. 1983, ApJ, 271, 575

Christiansen, W. N., Frater, R. H., Watkinson, A., et al. 1977, MNRAS, 181, 183

Dressler, A., Faber, S. M., Burstein, D., et al. 1987, ApJ, 313, L37

Feretti, L., Giovannini, G., Govoni, F., \& Murgia, M. 2012, A\&ARv, 20, 54

Garon, A. F., Rudnick, L., Wong, O. I., et al. 2019, AJ, 157, 126

Hardcastle, M. J., Croston, J. H., Shimwell, T. W., et al. 2019, MNRAS, 488, 3416

Heyvaerts, J., \& Norman, C. 1989, ApJ, 347, 1055

Jones, P. A., \& McAdam, W. B. 1996, MNRAS, 282, 137

Jonas, J., \& MeerKAT Team. 2016, in MeerKAT Science: On the Pathway to the SKA, 1

Kenyon, J. S., Smirnov, O. M., Grobler, T. L., \& Perkins, S. J. 2018, MNRAS, 478, 2399
Lane, W. M., Kassim, N. E., Ensslin, T. A., Harris, D. E., \& Perley, R. A. 2002, AJ, 123, 2985

Leahy, J. P. 1993, in DRAGNs, eds. H. J. Röser, \& K. Meisenheimer, 421, 1 Maccagni, F. M., Murgia, M., Serra, P., et al. 2020, A\&A, 634, A9

Makhathini, S. 2018, Ph.D. Thesis, Rhodes University, Drosty Rd, Grahamstown, 6139, Eastern Cape, South Africa

Mauch, T., Murphy, T., Buttery, H. J., et al. 2003, MNRAS, 342, 1117

Mauch, T., Cotton, W. D., Condon, J. J., et al. 2020, ApJ, 888, 61

McMullin, J. P., Waters, B., Schiebel, D., Young, W., \& Golap, K. 2007, in Astronomical Data Analysis Software and Systems XVI, eds. R. A. Shaw, F. Hill, \& D. J. Bell, ASP Conf. Ser., 376, 127

Missaglia, V., Massaro, F., Capetti, A., et al. 2019, A\&A, 626, A8

Offringa, A. R., \& Smirnov, O. 2017, MNRAS, 471, 301

Offringa, A. R., de Bruyn, A. G., Biehl, M., et al. 2010, MNRAS, 405, 155

Offringa, A. R., McKinley, B., Hurley-Walker, N., et al. 2014, MNRAS, 444, 606

Owen, F. N., \& Rudnick, L. 1976, ApJ, 205, L1

Owen, F. N., O’Dea, C. P., Inoue, M., \& Eilek, J. A. 1985, ApJ, 294, L85

Owen, F. N., Rudnick, L., Eilek, J., et al. 2014, ApJ, 794, 24

Perley, R. A., Dreher, J. W., \& Cowan, J. J. 1984, ApJ, 285, L35

Perucho, M., \& Martí, J. M. 2007, MNRAS, 382, 526

Pinkney, J. 1995, Ph.D. Thesis, New Mexico State University, USA

Ryle, M., \& Windram, M. D. 1968, MNRAS, 138, 1

Sakelliou, I., \& Merrifield, M. R. 2000, MNRAS, 311, 649

Schilizzi, R. T., \& McAdam, W. B. 1975, MmRAS, 79, 1

Sebastian, B., Lal, D. V., \& Pramesh Rao, A. 2017, AJ, 154, 169

Serra, P., Westmeier, T., Giese, N., et al. 2015, MNRAS, 448, 1922

Shin, J., Woo, J.-H., \& Mulchaey, J. S. 2016, ApJS, 227, 31

Sijbring, D., \& de Bruyn, A. G. 1998, A\&A, 331, 901

Sun, M. 2009, ApJ, 704, 1586

Woudt, P. A., Kraan-Korteweg, R. C., Lucey, J., Fairall, A. P., \& Moore, S. A. W. 2008, MNRAS, 383, 445 